\title{
公HAD
}

DOI: http://doi.org/10.22585/hospdomic.v2i4.53

\section{Resumen Ejecutivo del tratamiento antibiótico domiciliario endovenoso: Directrices de la Sociedad Española de Enfermedades Infecciosas y la Sociedad Española de Hospitalización a Domicilio*}

Executive summary of outpatient parenteral antimicrobial therapy: Guidelines of the Spanish Society of Clinical Microbiology and Infectious Diseases and the Spanish Domiciliary Hospitalisation Society*

*Este documento es la traducción al español del documento publica con anterioridad en la revista Enfermedades Infecciosas y Microbiología Clínica (1).

* Los datos complementarios asociados con este artículo se pueden encontrar, en la versión original en línea, en https://doi.org/10.1016/j.eimc.2018.03.012 (1).

Luis Eduardo López Cortés (Coordinador)', Abel Mujal Martínez (Coordinador)², Magdalena Fernández Martínez de Mandojana ${ }^{3}$, Natalia Martín ${ }^{4}$, Mercè Gil Bermejo ${ }^{5}$, Joan Solá Aznar 6 , Eulalia Villegas Bruguera 7 , Maria José Peláez Cantero ${ }^{8}$, Pilar Retamar Gentil', Miriam Delgado Vicente9, Víctor José González-Ramallo10, Miguel Ángel Ponce González"11, Manuel Mirón Rubio'2, $M^{a}$ Montserrat Gómez Rodríguez de Mendarozqueta ${ }^{13}$, Miguel Ángel Goenaga Sánchez ${ }^{14}$, Pedro Sanroma Mendizábal15, Elena Delgado Mejía ${ }^{16}$, Marcos Pajarón Guerrero ${ }^{15}$; en nombre de la Sociedad Española de Enfermedades Infecciosas y Microbiología Clínica (SEIMC) y de la Sociedad Española de Hospitalización a Domicilio (SEHAD).

1. Hospital Universitario Virgen Macarena, Instituto de Biomedicina de Sevilla (IBiS), Unidad Clínica de Enfermedades Infecciosas y Microbiología. Universidad de Sevilla, Centro Superior de Investigaciones Científicas. Sevilla, España.

2. Hospital de Sabadell, Corporació Sanitària Parc Tauli, Departamento de Medicina Interna, Unidad de Hospitalización a Domicilio. Universitat Autònoma. Barcelona, España.

3. Organización Sanitaria Integrada (OSI). Debabarrena, España.

4. Hospital San Pedro. Logroño, España.

5. Hospital Universitario de Bellvitge. Hospitalet del Llobregat, Barcelona, España.

6. Parc Taulí Hospital Universitari. Sabadell, Barcelona, España.

7. Hospital Dos de Maig. Barcelona, España.

8. Hospital Materno-Infantil Carlos Haya. Málaga, España.

9. Hospital Universitario. Álava, España.

10. Hospital General Universitario Gregorio Marañón. Madrid, España.

11. Hospital Universitario de Gran Canaria Dr. Negrín, Las palmas de Gran Canaria, España.

12. Hospital de Torrejón. Madrid, España.

13. Hospital Universitário. Álava, España.

14. Organización Sanitaria Integrada (OSI). Donostialdea, España.

15. Hospital Marqués de Valdecilla. Santander, España.

16. Hospital Son Espases. Palma de Mallorca, España. 
Otros autores de REIPI/SEHAD

Carmen Garde Orbaiz (Hospital Universitario Donostia), Mario García Lezcano (Hospital de Can Misses, lbiza), Andima Basterretxea Ozamiz (Hospital Universitario Cruces, Vizcaya), María Victoria Gil Navarro (Hospital Universitario Virgen del Rocío, Sevilla), Sara Ortonobés Roig (Parc Taulí Hospital Universitari, Sabadell, Barcelona), Eva Llobet Barberí (Hospital Dos de Maig, Barcelona), Francisca Sánchez Martínez (Hospital del Mar, Barcelona), Manuel Mirón Rubio (Hospital Universitario de Torrejón, Madrid), y Manuel del Río Vizoso (Hospital Son Espases, Palma de Mallorca).

\section{Correspondencia/Correspondence \\ Luis Eduardo López Cortés \\ luiselopezcortes@gmail.com}

\section{Recibido/Received}

08.08 .2018

\section{Aceptado/Accepted}

24.09.2018

\section{Conflicto de Intereses/Competing interest}

LELC ha realizado conferencias para Merck, Sharp and Dohme, y Angellini, ha recibido soporte para la investigación de Novartis, y ha sido formador para Merck, Sharp and Dohme. PRG ha sido miembro de consejos asesores y formador para Merck, Sharp and Dohme y Roche.

El resto de autores declara no tener conflicto de intereses.

\section{Agradecimientos/Acknowledgments}

Agradecemos la ayuda prestada en la realización y revisión redacción de este documento a Carlos Cervera, Rafael Luque, Laura Álvarez, José Miguel Cisneros y Miguel Ángel Goenaga.

Con el apoyo del Plan Nacional de I+D+i y del Instituto de Salud Carlos III, Subdirección General de Redes y Centros de Investigación Cooperativa, Ministerio de Economía y Competitividad, Red Española de Investigación en Patología Infecciosa (REIPI RD 12/0015) - cofinanciado por el Fondo Europeo de Desarrollo Regional "Una forma de hacer Europa" FEDER.

\section{CÓMO CITAR ESTE TRABAJO | HOW TO CITE THIS PAPER}

López Cortés LD, Mujal Martínez A, Fernández Martínez de Mandojana M, Martín N, Gil Bermejo M, Solá Aznar J, et al; Grupo de la Sociedad Española de Enfermedades Infecciosas y Microbiología Clínica (SEIMC), Grupo de la Sociedad Española de Hospitalización a Domicilio (SEHAD). Resumen Ejecutivo del tratamiento antibiótico domiciliario endovenoso: Directrices de la Sociedad Española de Enfermedades Infecciosas y la Sociedad Española de Hospitalización a Domicilio. Hosp Domic. 2018;2(4): 165-77. 


\section{RESUMEN}

Los programas de tratamiento antibiótico domiciliario endovenoso (TADE) permiten iniciar o completar el tratamiento antimicrobiano por vía endovenosa de prácticamente cualquier tipo de infección en el domicilio, siempre y cuando se realice una selección del paciente acorde al tipo de programa de TADE que se dispone. Aunque hay aspectos del manejo clínico de las infecciones en el domicilio que son superponibles en la mayoría de los casos a la realizada en la hospitalización convencional (selección de la antibioterapia, duración del tratamiento, etc.), existen numerosos aspectos que son específicos de esta modalidad asistencial. Resulta imprescindible conocerlos para que el TADE siga siendo igual de eficaz y seguro que la hospitalización convencional. El objetivo de esta guía clínica es por tanto proporcionar recomendaciones basadas en la evidencia realizadas por expertos para homogeneizar la práctica clínica de esta modalidad asistencial y contribuir a que se incremente progresivamente el número de pacientes que pueden ser atendidos y recibir tratamiento endovenoso en su propio domicilio.

Palabras clave: Antibacterianos; Programas de Optimización del Uso de los Antimicrobianos; Administración Intravenosa; Terapia de Infusión a Domicilio; Servicios de Atención a Domicilio Provisto por Hospital.

\section{ABSTRACT}

Outpatient parenteral antimicrobial therapy (OPAT) programmes make it possible to start or complete intravenous antimicrobial therapy for practically any type of infection at home, provided that patient selection is appropriate for the type of OPAT programme available. Although the clinical management of infections in the home setting is comparable in many respects to that offered in conventional hospitalization (selection of antibiotics, duration of treatment, etc.), there are many aspects that are specific to this care modality. It is essential to be aware of them so that OPAT continues to be as safe and effective as inpatient care. The objective of this clinical guideline is therefore to provide evidence-and expert-based recommendations with a view to standardizing clinical practice in this care modality and contribute to a progressive increase in the number of patients who can be cared for and receive intravenous therapy in their own homes.

Keywords: Anti-Bacterial Agents; Antimicrobial Stewardship; Administration, Intravenous; Home Infusion Therapy; Home Care Services, Hospital-Based. 


\section{INTRODUCCIÓN}

EI TADE (Tratamiento Antibiótico Domiciliario Endovenoso) es una modalidad asistencial en la cual se trata al paciente en su domicilio con fármacos por vía intravenosa. El término fue acuñado por Rucker et al. en 1974 (2), en una publicación sobre antibioterapia administrada de forma parenteral a niños con fibrosis quística pero que pernoctaban fuera del hospital. La primera experiencia en pacientes adultos fue descrita por Antoniskis et al. en 1978 (3), en trece pacientes principalmente con osteomielitis que se autoadministraron el antibiótico por vía parenteral. Progresivamente los programas TADE se han ido desarrollando en diferentes países como Canadá, Australia, Nueva Zelanda, Singapur, Italia, Irlanda, Reino Unido, Bélgica y España, mostrando ventajas tanto para los pacientes como para el sistema sanitario. EI TADE ha demostrado ser una modalidad asistencial segura, efectiva y más eficiente que la hospitalización convencional en el tratamiento de enfermedades infecciosas de muy diferente índole. La experiencia en los programas TADE ha ido creciendo y evolucionando como se constata al revisar la literatura: la reducción de las estancias hospitalarias a favor de la duración del TADE, el inicio del tratamiento antibiótico sin hospitalización previa, el incremento del uso de catéteres centrales de inserción periférica (CCIP) para tratamientos prolongados, así como la atención a pacientes pediátricos o de edad avanzada. En nuestro país se ha desarrollado de forma heterogénea dependiente de múltiples factores influyentes como el tipo de necesidades de cada uno de los servicios hospitalarios, los recursos de cada centro y sobre todo el conocimiento y la decisión de las propias consejerías de Sanidad de incluir o no esta modalidad asistencial en los hospitales de cada comunidad autónoma. Los aspectos clave de un programa de TADE son: 1 definir la estructura del programa y los componentes del equipo, 2 seleccionar de forma adecuada a los pacientes, 3 el manejo y la forma de administrar cada uno de los antimicrobianos, 4 la monitorización de los pacientes durante el proceso, y 5 el análisis de los resultados y una gestión clínica adecuada.

La presente declaración se escribió siguiendo las directrices de Sociedad Española de Enfermedades Infecciosas y Microbiología Clínica (SEIMC) para declaraciones de consenso (www. seimc.org), así como las recomendaciones de Agree Collaboration (www.agreecollaboration.org) para evaluar la calidad metodológica de las guías de práctica clínica. En varias reuniones, los autores seleccionaron un conjunto de preguntas diseñadas para formar la base del documento. Sus recomendaciones se basan en una revisión crítica sistemática de la literatura que incluye, cuando es necesario, la opinión de expertos, que son miembros de la Sociedad Española de Enfermedades Infecciosas y Microbiología Clínica (SEIMC) y de la Sociedad Española de Hospitalización a Domicilio (SEHAD). Sus recomendaciones se han ajustado de acuerdo con la evidencia científica disponible (ver DOI: https://doi.org/10.1016/j.eimc.2018.03.012 (1)). Todos los autores y los coordinadores de la declaración han acordado los contenidos y las conclusiones del documento. Antes de la publicación final, el manuscrito estaba disponible en línea para que todos los miembros de SEIMC lo leyeran y para hacer comentarios y sugerencias. 


\section{DEFINICIÓN DE TADE. COMPOSICIÓN DE UN EQUIPO TADE. CIRCUITO ASISTENCIAL DEL PROGRAMA TADE}

\section{Recomendaciones:}

- La administración de un antibiótico parenteral en un paciente ambulatorio (TADE) es una práctica cada vez más frecuente, segura, efectiva y con menor coste (A-II).

- Gran variedad de infecciones son candidatas a TADE (A-III).

- El paciente susceptible de esta terapia puede ser derivado desde cualquier nivel asistencial (B-II)

- Debe ser un programa multidisciplinar: personal médico, de enfermería y administrativo adscrito al programa, en estrecha colaboración con farmacéuticos y microbiólogos (A-II).

- Se necesitan guías de recomendación y supervisión adecuada para garantizar la idoneidad y seguridad del tratamiento (A-II).

\section{ROL DE CADA MIEMBRO DEL EQUIPO TADE. CRITERIOS DE INCLUSIÓN DE UN PACIENTE EN UN PROGRAMA TADE}

\section{Recomendaciones:}

- El equipo asistencial para un programa de TADE en HAD es, por sus características especiales, multidisciplinar, y está compuesto por personal médico, de enfermería, farmacéuticos, auxiliares y por el paciente y su cuidador (A-III).

- El factor fundamental para garantizar el éxito de un programa TADE es la adecuada selección de los pacientes (A-III).

- Para que el TADE sea seguro y efectivo, el paciente tiene que estar en situación de estabilidad clínica y hemodinámica y cumplir los criterios de ingreso generales y específicos de inclusión en un programa TADE (A-III).

- Las preguntas de High resumen y valoran la adecuación del ingreso en un programa TADE (A-III). 


\section{MODALIDADES DE ADMINISTRACIÓN DE ANTIMICROBIANOS EN TADE Y ACCESOS VENOSOS. COMPLICACIONES DE LOS ACCESOS VENOSOS}

\section{Recomendaciones:}

- Un elemento clave para el éxito del TADE radica en la acertada elección de la modalidad de administración, así como del tipo de acceso venoso, en función del antimicrobiano a administrar, duración del tratamiento y de las características y habilidades del paciente/ cuidador (A-III).

- La autoadministración se ha demostrado una forma segura y eficaz en el TADE y facilita la asociación simultánea de dos o más antibióticos (A-II).

- Si el antimicrobiano tiene estabilidad de varios días entre $2-8^{\circ} \mathrm{C}$, es factible la preparación de varias dosis con anticipación y su conservación en nevera. En estos casos, se aconseja la preparación de los dispositivos de infusión en cámara de flujo laminar para asegurar la estabilidad microbiológica de la dilución (A-III).

- La adecuada elección del acceso venoso es clave para el éxito del TADE y dependerá de las características del fármaco a infundir, número de dosis diarias, duración del tratamiento y las características propias del paciente (A- III).

- Los catéteres venosos periféricos, tanto cortos como de línea media, no están recomendados para la administración de terapias vesicantes o con osmolaridad $>500-600$ mOsm y/o $\mathrm{pH}<5$ ó $>9$ (A-III).

- Los CVP cortos se recomiendan cuando la duración prevista del TADE sea inferior a 7 días. (B-III).

- Los CVP de línea media se recomiendan en TADE de duración entre 7 y 14 días. (B-III).

- Los catéteres venosos centrales, ya sean catéteres centrales de inserción periférica (CCIP) o de inserción central se recomiendan para la administración de terapias vesicantes o con osmolaridad >500-600 mOsm y/o pH <5 ó >9 (A-III)

- Los CCIP se recomiendan cuando la duración prevista del TADE sea superior a 15 días. (B-III).

- Se desaconseja el uso de catéteres centrales de inserción periférica en pacientes con insuficiencia renal crónica estadio 3b (Filtrado Glomerular $<45 \mathrm{ml} / \mathrm{min}$ ) y que sean posibles candidatos a hemodiálisis (A-II).

- Se desaconseja asimismo el acceso de vena subclavia en pacientes con insuficiencia renal crónica estadio 3b (Filtrado Glomerular $<45 \mathrm{ml} / \mathrm{min}$ ) y que sean posibles candidatos a hemodiálisis (A-I).

- Si recomienda que el recambio del catéter venoso periférico corto se haga según criterio clínico o de forma programada cada 72-96 horas con la finalidad de reducir las tasas de infección o flebitis (B-I).

- En el resto de catéteres, incluidos los de línea media, sólo se aconseja su recambio por criterios clínico (A-III)

- Las complicaciones relacionadas con el uso de catéteres en TADE son similares a las reportadas en el uso hospitalario y en pocas ocasiones obliga al reingreso del paciente (A-III). 


\section{CARACTERÍSTICAS FARMACOCINÉTICAS, FARMACODINÁMICAS Y ESTABILIDAD DE LOS FÁRMACOS ADMINISTRADOS EN TADE. CRITERIOS DE ELECCIÓN DEL ANTIMICROBIANO. ASOCIACIÓN SIMULTÁNEA DE ANTIBIÓTICOS EN TADE}

\section{Recomendaciones:}

- Las propiedades farmacocinéticas y farmacodinámicas del antimicrobiano a infundir en un programa TADE van a condicionar la modalidad de infusión (manual o mecánica) y la elección del acceso vascular (B-II).

- Si el antimicrobiano no se va a administrar de forma inmediata es recomendable preparar la dilución en campana de flujo laminar (B-II)

- La autoadministración del antimicrobiano por parte del paciente o cuidador es un método seguro y permite realizar TADE complejo (múltiples dosis y múltiples antibióticos) y más eficiente (B-II).

- Las bombas de infusión electrónicas son dispositivos útiles en caso de administración de antimicrobianos en múltiples dosis (B-II)

\section{MONITORIZACIÓN DE LOS PACIENTES EN TADE. INTERACCIONES FARMACOLÓGICAS. EFECTOS ADVERSOS. MANEJO DE LAS COMPLICACIONES DEL TADE}

\section{Recomendaciones:}

- Los efectos secundarios del TADE son relativamente frecuentes, y pueden obligar al retorno al centro hospitalario, por lo que los circuitos de detección y respuesta de los profesionales ante dichas complicaciones deben ser ágiles y estar claramente protocolizados (B-II).

- La monitorización debe individualizarse atendiendo al perfil clínico del paciente y su entorno cuidador, a las características del fármaco y a su forma de administración (A-III).

- Todo TADE debe realizarse siguiendo las guías y recomendaciones de las sociedades médicas relevantes, debe incluir una adecuada vigilancia clínica y analítica, y debe quedar correctamente registrada en la documentación del episodio (A-II).

- Los profesionales deben conocer las interacciones farmacológicas del TADE, ya que pueden provocar el aumento o disminución de la concentración de los medicamentos, los cambios en la efectividad de los fármacos en interacción (disminuyéndola o aumentándola), y/o la gravedad de sus efectos secundarios (A-II). 
- Se recomienda la monitorización de las reacciones adversas medicamentosas previsibles (A-II). Por ejemplo, la realización de niveles valle de gentamicina para evitar la nefrotoxicidad o la determinación de la creatinin-fosfoquinasa durante la administración de daptomicina para reducir el riesgo de rabdomiolisis grave.

- Los profesionales de los equipos TADE deben proveerse del tratamiento necesario para afrontar una reacción adversa medicamentosa potencialmente grave en el domicilio según el riesgo conocido de la misma (corticoides en los betalactámicos, anticonvulsivantes con los carbapenems entre otros) (A-III).

- Es aconsejable realizar controles analíticos semanales que incluyan un hemograma, función renal y hepática. (B-II).

\section{CONSIDERACIONES DE TADE EN PEDIATRÍA}

\section{Recomendaciones:}

- EI TADE en pediatría proporciona mejor calidad de vida y mayor satisfacción para los pacientes y sus familias (A-I).

- EI TADE en pediatría es efectivo y seguro en pacientes previamente seleccionados (B-II).

- La elección del antimicrobiano para TADE en pediatría debe seguir los mismos conceptos (farmacocinética, eficacia y seguridad) que para el paciente adulto (B-II).

- Los catéteres venosos periféricos cortos se recomiendan cuando la duración prevista del TADE sea inferior a 7 días. (B-III).

- Los catéteres venosos periféricos de línea media se recomiendan en TADE de duración entre 7 y 14 días. (B-III).

- Los catéteres venosos de inserción periférica se recomiendan cuando la duración prevista del TADE sea superior a 15 días. (B-III).

- Aunque la administración del antimicrobiano la realiza habitualmente personal de enfermería, tras un adecuado adiestramiento, puede ser llevada a cabo también por los cuidadores del paciente, de una forma eficaz y segura (C-III).

\section{PROGRAMAS DE OPTIMIZACIÓN DE USO DE ANTIMICROBIANOS Y PROGRAMAS TADE: UNA RELACIÓN NECESARIA}

\section{Recomendaciones:}

- Se recomienda la pertenencia de uno de los responsables del grupo TADE al programa de optimización de antibioterapia (PROA) del hospital (C-III).

- Se recomienda que la selección y forma de uso de los antimicrobianos en los programas TADE, se adapte a las directrices del PROA de referencia (C-III) 
- Los TADE facilitan la optimización del uso de los antimicrobianos en situaciones empíricas en las que no es posible o deseable la utilización de la vía oral o en tratamientos dirigidos cuando no existen alternativas orales efectivas (B-II).

- Los TADE deben seguir los criterios utilizados en los PROA para la terapia secuencial (BII).

- La duración total del tratamiento debe planificarse en base a las evidencias disponibles (A-I) y establecer medidas de evaluación y control para el cumplimiento de la misma (C-III).

\section{EVIDENCIA DE LA EFICACIA Y SEGURIDAD DE TADE EN INFECCIÓN URINARIA}

\section{Recomendaciones:}

- Las pautas de tratamiento antimicrobiano de las infecciones urinarias, así como su duración en régimen de TADE deben ser las mismas que para los pacientes en hospitalización convencional (C-III).

- La utilización de ertapenem en pacientes con ITU por enterobacterias productoras de betalactamasas de espectro extendido (BLEE) en régimen de TADE ha demostrado ser una opción coste-efectiva (B-III).

\section{EVIDENCIA DE LA EFICACIA Y SEGURIDAD DE TADE EN INFECCIÓN DE PIEL Y PARTES BLANDAS}

\section{Recomendaciones:}

- Las pautas de tratamiento antimicrobiano de las infecciones de piel y partes blandas, así como su duración en régimen de TADE deben ser las mismas que para los pacientes en hospitalización convencional (C-III)

- Es factible el tratamiento de formas moderadas y severas de las infecciones de piel y partes blandas mediante programas TADE con criterios de seguridad, efectividad y eficiencia (B-II)

- Numerosos pacientes con diagnóstico de celulitis pueden ser derivados directamente desde los Servicios de Urgencias para seguir TADE tras un periodo de observación e inicio de tratamiento antibiótico empírico. En estos pacientes, la presencia de fiebre y recuento leucocitario mayor de 15.000 células se relaciona con una mayor tasa de fracaso del TADE y por consiguiente con un mayor retorno al hospital (B-III)

- Se recomienda un control clínico más estrecho de las de las infecciones de piel y partes blandas en pacientes de sexo femenino, diabéticos y en TADE con teicoplanina ya que en algún estudio se han mostrado como factores independientes de fracaso del TADE (B-III) 


\section{EVIDENCIA DE LA EFICACIA Y SEGURIDAD DE TADE EN ENDOCARDITIS INFECCIOSA}

\section{Recomendaciones:}

- Los pacientes con El a los que se le proponga TADE se les deberá haber completado un estudio y estabilizado en hospitalización convencional previamente a ello (C-I)

- Deberán tener una baja probabilidad de complicaciones, las más frecuentes de las cuales son el desarrollo de insuficiencia cardiaca y de embolismos sistémicos (C-I)

- Para ello deberán haber cumplido al menos dos semanas de tratamiento correcto en hospitalización convencional (7-10 días en el caso de Streptococcus del grupo viridans), con estabilidad clínica y hemodinámica y con hemocultivos de control negativos (B-II)

- Para otras formas de endocarditis diferentes a la anterior, la decisión de incluir al paciente para un programa de TADE deberá ser siempre consensuada con el equipo médicoquirúrgico del paciente (C-III)

- La duración del tratamiento antibiótico en régimen de TADE será la misma que se aconseja para el paciente hospitalizado (B-II)

- Para el tratamiento de la endocarditis por Streptococcus del grupo viridans en TADE se aconseja ceftriaxona (A-I)

- Para la endocarditis por Staphylococcus aureus meticilin sensible, se aconseja siempre que sea posible cloxacilina (C-III)

- Los pacientes con El por SAMR, así como las producidas por Enterococcus sp. es difícil que puedan cumplir criterios de TADE. En estos casos, su inclusión en el programa de TADE dependerá de la experiencia del equipo y siempre será consensuada. (C-III)

\section{EVIDENCIA DE LA EFICACIA Y SEGURIDAD DE TADE EN INFECCIÓN RESPIRATORIA Y NEUMONÍA}

\section{Recomendaciones:}

- EI TADE es seguro y efectivo en el tratamiento de las infecciones respiratorias (B-II)

- Las pautas de tratamiento antimicrobiano para las infecciones respiratorias deben ser las mismas que en pacientes hospitalizados convencionalmente incluyendo los antibióticos antipseudomónicos (C-III).

- No hay diferencias de seguridad y efectividad en el tratamiento de infecciones respiratorias con TADE: EPOC agudizado, neumonía de la comunidad, fibrosis quística y bronquiectasias no-FQ (B-II).

- Existe escasa evidencia de infecciones respiratorias tipo absceso pulmonar y empiema pleural tratado con TADE (C-III)

- La duración del TADE en infecciones respiratorias será la misma que la aconsejada en los pacientes hospitalizados convencionalmente (B-II) 
- EI TADE en infecciones respiratorias por Pseudomonas aeruginosa, incluyendo las cepas multirresistentes, es seguro y efectivo (B-II).

- Los pacientes respiratorios crónicos deben continuar con las terapias respiratorias a domicilio necesarias (oxigenoterapia, aerosolterapia y ventilación no invasiva domiciliaria) así como otros tratamientos habituales en las agudizaciones respiratorias tales como esteroides intravenosos $\mathrm{U}$ orales (A-I)

\section{EVIDENCIA DE LA EFICACIA Y SEGURIDAD DE TADE EN INFECCIÓN INTRAABDOMINAL, ABSCESOS INTRAABDOMINALES E INFECCIONES DE LA VÍA BILIAR}

\section{Recomendaciones:}

- Cualquier infección intraabdominal puede tratarse en régimen de TADE cuando se cumplen los requisitos para prestar atención sanitaria según este modelo asistencial (C-III)

- La mayoría de las infecciones intraabdominales requieren un periodo previo de hospitalización convencional antes del tratamiento en TADE. Sólo la diverticulitis no complicada puede ser tratada directamente desde Urgencias. (B-III)

- Las pautas de tratamiento antimicrobiano de la infección intraabdominal así como su duración en régimen de TADE deben ser las mismas que para los pacientes en hospitalización convencional (C-III)

\section{EVIDENCIA DE LA EFICACIA Y SEGURIDAD DE TADE EN INFECCIÓN OSTEOARTICULAR}

\section{Recomendaciones:}

- El tratamiento de las infecciones osteoarticulares requiere en ocasiones de la combinación de tratamiento quirúrgico y tratamiento antibiótico (A-II)

- El tratamiento parenteral seguido de tratamiento antibiótico oral es tan eficaz como el tratamiento parenteral a largo plazo (B-III)

- Se aconseja una coordinación estrecha con los servicios de traumatología y enfermedades infecciosas (B-III).

- Las pautas de tratamiento antimicrobiano de las infecciones osteoarticulares así como su duración en régimen de TADE deben ser las mismas que para los pacientes en hospitalización convencional (C-III)

- Cuando se realiza correctamente, el TADE ofrece mayor comodidad para el paciente, disminuye el riesgo de infecciones nosocomiales y reduce el costo para el sistema de salud (B-III). 


\section{EVIDENCIA DE LA EFICACIA Y SEGURIDAD DE TADE EN INFECCIONES DEL SISTEMA NERVIOSO CENTRAL}

\section{Recomendaciones:}

- Las infecciones del sistema nervioso central pueden ser tratadas con seguridad en el domicilio del paciente, de manera que ciertas meningitis bacterianas, meningoencefalitis herpéticas o abscesos cerebrales pueden finalizar el tratamiento mediante TADE sin añadir un mayor número de complicaciones que las de la hospitalización convencional con un buen resultado clínico (C-III).

- Las pautas de tratamiento antimicrobiano de las infecciones del sistema nervioso central, así como su duración en régimen de TADE deben ser las mismas que para los pacientes en hospitalización convencional (C-III)

\section{EVIDENCIA DE LA EFICACIA Y SEGURIDAD DE TADE EN INFECCIONES EN PACIENTES CON NEUTROPENIA FEBRIL}

\section{Recomendaciones:}

- El TADE en pacientes neutropénicos es efectivo, seguro y eficiente, incluso en pacientes pediátricos (B-III)

- EI TADE en pacientes con neutropenia febril mejora los parámetros de calidad de vida con respecto a los pacientes ingresados en hospitalización convencional (B-II).

- Las pautas de tratamiento antimicrobiano en la neutropenia febril, así como su duración en régimen de TADE deben ser las mismas que para los pacientes en hospitalización convencional (C-III)

\section{EVIDENCIA DE LA EFICACIA Y SEGURIDAD DE TADE EN INFECCIONES POR BACTERIAS MULTIRRESISTENTES}

\section{Recomendaciones:}

- El TADE se ha evidenciado como efectivo y seguro en las infecciones por bacterias multirresistentes (A-II) 
- Las pautas de tratamiento antimicrobiano en las infecciones por bacterias multirresistentes, así como su duración en régimen de TADE deben ser las mismas que para los pacientes en hospitalización convencional (C-III)

- Durante el periodo de tratamiento se deben guardar las medidas habituales de higiene, control y prevención del microorganismo, siendo la higiene de manos la base de las medidas a adoptar en domicilio (A-II).

- La administración domiciliaria de meropenem requiere de la participación del propio paciente o su cuidador, así como su adiestramiento en el manejo de dispositivos de infusión portátiles dada la inestabilidad de este antibiótico a temperatura ambiente (A-II)

- Daptomicina supone una opción idónea para el TADE como alternativa a vancomicina debido a su posología de administración única diaria, no precisar monitorización de niveles plasmáticos y menor tasa de efectos adversos que la vancomicina en relación a nefrotoxicidad principalmente (B-II)

\section{EVIDENCIA DE LA EFICACIA Y SEGURIDAD DE TADE EN INFECCIONES ASOCIADAS A TERAPIA BIOLÓGICA:}

\section{Recomendaciones:}

- Los pacientes con procesos infecciosos relacionados con agentes biológicos deben de reunir criterios de estabilidad clínica y hemodinámica antes de realizar TADE (B-II).

- Las pautas de tratamiento antimicrobiano en las infecciones asociadas a terapia biológica, así como su duración en régimen de TADE deben ser las mismas que para los pacientes en hospitalización convencional (C-III).

- En el caso del TADE en infecciones víricas, infecciones fúngicas, así como en aquellas causadas por microorganismos oportunistas, se seguirán las recomendaciones terapéuticas recogidas en las guías existentes al respecto (C-III)

\section{BIBLIOGRAFÍA}

1. López Cortés LD, Mujal Martínez A, Fernández Martínez de Mandojana M, Martín N, Gil Bermejo M, Solá Aznar J, et al; Grup of the Sociedad Española de Enfermedades Infecciosas y Microbiología Clínica (SEIMC), Grup of the Sociedad Española de Hospitalización a Domicilio (SEHAD). Executive summary of outpatient parenteral antimicrobial therapy: Guidelines of the Spanish Society of Clinical Microbiology and Infectious Diseases and the Spanish Domiciliary Hospitalisation Society. Enferm Infecc Microbiol Clin. 2018. DOI: 10.1016/j.eimc.2018.03.012; PMID: 29784453

2. Rucker RW, Harrison GM. Outpatient intravenous medications in the management of cystic fibrosis. Pediatrics. 1974;54(3):358-60. PMID: 4213282

3. Antoniskis A, Anderson BC, Van Volkinburg EJ, Jackson JM, Gilbert DN. Feasibility of outpatient self-administration of parenteral antibiotics. West J Med. 1978;128(3):203-6. PMID: 636409 